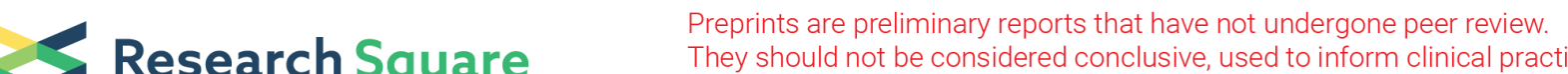 or referenced by the media as validated information.
}

\section{Mathematical Model for COVID-19 Management in Crowded Settlements and High-Activity Areas}

\author{
Amos Ssematimba \\ Gulu University \\ Juliet Nakakawa ( $\nabla$ nakakawa@cns.mak.ac.ug ) \\ Makerere University \\ Joseph Ssebuliba \\ Makerere University \\ Joseph Y. T Mugisha \\ Makerere University
}

\section{Research Article}

Keywords: Mathematical modelling, standard operating procedures , COVID-19, crowding, critical area

Posted Date: December 3rd, 2020

DOl: https://doi.org/10.21203/rs.3.rs-85312/v2

License: (9) This work is licensed under a Creative Commons Attribution 4.0 International License. Read Full License

Version of Record: A version of this preprint was published at International Journal of Dynamics and Control on March 13th, 2021. See the published version at https://doi.org/10.1007/s40435-021-00781-9. 


\title{
Mathematical Model for COVID-19 Management in Crowded Settlements and High-Activity Areas
}

\author{
A. Ssematimba · J.N. Nakakawa · J. Ssebuliba · J.Y.T \\ Mugisha
}

Received: date / Accepted: date

\begin{abstract}
This paper develops and analyses a population density-dependent mathematical model to study the transmission dynamics of COVID-19 in crowded settlements such as refugee camps, schools, markets and churches. The model quantifies the potential impact of physical/social distancing and population density on the disease burden. Results reveal that with no fatalities and no infected entrants, the reproduction numbers associated with asymptomatic and symptomatic cases are inversely proportional to; the habitat area size, and the efforts employed in tracing and hospitalising these cases. The critical habitat area below which the disease dies out is directly proportion to the time taken to identify and hospitalise infected individuals. Results also show that disease persistence in the community is guaranteed even with minimal admission of infected individuals. Our results further show that as the level of compliance to standard operating procedures (SOPs) increases, then the disease prevalence peaks are greatly reduced and delayed. Therefore, proper adherence to SOPs such as use of masks, physical distancing measures and effective contact tracing should be highly enforced in crowded settings if COVID-19 is to be mitigated.
\end{abstract}

Keywords Mathematical modelling $\cdot$ standard operating procedures · COVID-19 · crowding $\cdot$ critical area

Mathematics Subject Classification (2010) 34A34 - 34D23 - 92B05 - 91B70

\section{Introduction}

With over 16 million confirmed cases and 0.64 million deaths globally (as of $26^{\text {th }}$ July 2020) [14], the on-going coronavirus 2019 (COVID-19) pandemic is among the most devastating global human epidemics. Besides the public health impact, there is also the global economic devastation associated with the pandemic that renders its control a priority [15]. The disease can spread through direct and indirect contact with aerosols from infectious individuals and physical/social distancing and isolation are some of the recommended measures to curb its spread within communities.

Directly transmitted infections tend to increase with population densities with scaled frequency of contact rates [17]. Local public sites with extremely high population density such as transport hubs, markets, large social, political or religion mass gatherings and business locations are regarded as high risk transmission points for highly respiratory infection diseases [17] such as COVID-19 due to close contact between hosts [18].

At the onset of implementation of possible control measures of COVID-19, lock-down of activities that were a source of crowding were effected. However when the disease mechanisms and dynamics begun to evolve, socialeconomic impacts dictated ease of lock-downs. The uncertainties associated with the timing and levels of effective ease the lock-downs, along with social-economic and political agitations required careful assessment to quantify

\footnotetext{
A. Ssematimba

Department of Mathematics, Faculty of Science, Gulu University, P.O. Box 166, Gulu, Uganda

E-mail: amos.ssematimba@gmail.com

J.N. Nakakawa

Department of Mathematics, School of Physical Sciences, College of Natural Science, Makerere University, P.0. Box 7062, Kampala, Uganda E-mail: nakakawa@cns.mak.ac.ug

J. Ssebuliba

Department of Mathematics, School of Physical Sciences, College of Natural Science, Makerere University, P.0. Box 7062, Kampala, Uganda E-mail: ssebulibaj@cns.mak.ac.ug

J.Y.T. Mugisha

Department of Mathematics, School of Physical Sciences, College of Natural Science, Makerere University, P.0. Box 7062, Kampala, Uganda E-mail: jytmugisha@cns.mak.ac.ug
} 
the dynamics of population dispersal especially for locations with extremely high densities. The efficacy of specific measures depends on population density and this should be considered during decision making process.

Mathematical models can be deployed to make efficacy assessments and such are formulated under specific assumptions pertaining to the force of infection, mixing patterns, diseases spread mechanisms and purpose of the study. The force of infection is driven by a contact rate whose expression fundamentally differ depending on whether a frequency or density dependent formulation is used. The latter assumes a constant rate while the former assumes a rate that depends on the total population size [6-11]. However, the explicit incorporation of habitat area size or population density in formulating the force of infection is not common yet it can be handy in addressing specific population density-related questions for diseases that spread via the aerosols and airborne route [7].

Given the transmission mechanisms of COVID-19, explicit incorporation of habitat area size in the formulation of the force of infection may add more realism in capturing the dynamics of the disease in crowded settlements such as refugee camps and high-activity areas like markets. This dependence can be modelled using compartmental models in which the contact rate explicitly depends on population density in the settlement $[7$, 13]. This explicit approach would enable the direct assessment of the impact of habitat area size and population density on the dynamics of the disease and thus guide the design of outbreak control measures suitable for crowded settlements.

In this study, we formulate and analyze a mathematical model in which we explicitly incorporate habitat area size in the force of infection together with processes that influence spread dynamics of COVID-19 in high population density settlements. We use the model to quantify the potential impact of physical/social distancing and population density on the disease burden. We further investigate the how the variation of density dependent transmission rates would influence adherence to SOPs. The rest of this paper is organised as follows; in Section 2, we present the model description, mathematical analysis is given in Section 3, numerical simulation in Section 4 and lastly a discussion in Section 6.

\section{Model Formulation}

\subsection{Model Description}

A compartmental SEIHR(S) epidemic model where $S(t)$ are the susceptibles, $E(t)$ are the latently infected, $\mathrm{I}(\mathrm{t})$ are the infectious individuals comprising of the asymptomatics $I_{a}(t)$ and symptomatics $I_{s}(t), H(t)$ are the hospitalized and $R(t)$ are the recovereds. The modelled processes/transitions are as follows: upon contact with infectious material, a susceptible individual becomes latently infected and remains in this state for the duration of the latent period, $1 / \rho$. Some individuals (a fraction, $c$ ) can be traced and quarantined and hospitalized before the end of latency while the rest proceed to either become asymptomatically (a fraction $r$ ) or symptomatically infectious for respective durations $\left(1 / \omega_{a}\right)$ and $\left(1 /\left(\omega_{s}+\sigma\right)\right)$, where $\sigma$, is the disease-induced death rate. After this duration, infectious individuals are then identified and hospitalized for a period $(1 /(\alpha+h \sigma))$ where $h$, is the reduction in disease-induced death rate for hospitalized individuals. Upon recovery, individuals are conferred a disease-induced immunity for a duration $1 / \tau$, which is pending further studies for appropriate calibration. The model captures recruitment, at a constant rate $\pi$, of individuals into the habitat with a fraction, $a$ being asymptomatically infected, $e$ being latently infected and the rest being susceptible. Susceptible and recovered individuals exit the habitat at a per-capita rate $\mu$. In this model, vital dynamics are not considered.

The formulation of the force of infection as $\left(\beta b S\left(m I_{a}+I_{s}+g H\right)\right) / A$, where $\beta$ is the transmission coefficient with dimension of area per time and can be interpreted as the effective area over which a susceptible makes contact in unit time [7], $b$ is the proportion of susceptible individuals, $m$ and $g$, are the percent reductions in infectivity of asymptomatic infectious and hospitalized individuals respectively and $A$, is the size of the area occupied by the population being studied. In formulating the transmission term, we assume (as done in [7,13]) that; the rate of contact between individuals increases directly with the density of the population, $N / A$, and the force of infection increases with the density of infectious individuals, $I / A$. 
2.2 Model equations

From the proceeding description, the dynamics of the model system are given as;

$$
\begin{aligned}
\frac{d S}{d t} & =(1-(e+a)) \pi-\frac{\beta b S\left(m I_{a}+I_{s}+g H\right)}{A}-\mu S+\tau R, \\
\frac{d E}{d t} & =e \pi+\frac{\beta b S\left(m I_{a}+I_{s}+g H\right)}{A}-\rho E, \\
\frac{d I_{a}}{d t} & =a \pi+r \rho E-\omega_{a} I_{a}, \\
\frac{d I_{s}}{d t} & =(1-(c+r)) \rho E-\sigma I_{s}-\omega_{s} I_{s}, \\
\frac{d H}{d t} & =c \rho E+\omega_{a} I_{a}+\omega_{s} I_{s}-h \sigma H-\alpha H, \\
\frac{d R}{d t} & =\alpha H-\mu R-\tau R .
\end{aligned}
$$

\section{Mathematical Analysis}

3.1 Basic properties of the model

The epidemiological feasibility of system (1) are guaranteed by proving that all its variables are non-negative at all time $t$, that is, the model's outputs with positive initial values will always remain positive at all time $t \geq 0$ in the following theorem.

Theorem 1 Suppose the model's initial values are $S(0) \geq 0, E(0) \geq 0, I_{a}(0) \geq 0, I_{s}(0) \geq 0, H(0) \geq 0$ and $R(0) \geq 0$, then the model's solutions, $\left(S(t), E(t), I_{a}(t), I_{s}(t), H(t), R(t)\right)$, with positive initial values will remain positive for all time $t \geq 0$.

Proof To prove positivity of the solution, it is sufficient to show that all the trajectories of system (1) are nonnegative for time $t>0$. From equation the first equation of system (1), the evolution of susceptible individuals over time will be given by the inequality;

$$
\frac{d S(t)}{d t} \geq-\left(\frac{\beta b\left(m I_{a}(t)+I_{s}(t)+g H(t)\right)}{A}+\mu\right) S(t)
$$

by solving the inequality and taking the limit as $t \rightarrow \infty$, we have

$$
\begin{aligned}
S(t) & \geq S_{0} \exp \left\{-\left(\mu t+\int_{0}^{t}\left(\frac{\beta b\left(m I_{a}(\tau)+I_{s}(\tau)+g H(\tau)\right)}{A}\right) d \tau\right)\right\}, \\
\lim _{t \rightarrow \infty} \inf S(t) \geq 0 . &
\end{aligned}
$$

Similarly, it can be shown that $E(t) \geq 0, I_{a}(t) \geq 0, I_{s}(t) \geq 0, H(t) \geq 0$ and $R(t) \geq 0$. Hence all solutions of system (1) will remain non-negative whenever we have non-negative initial conditions.

Theorem 2 The closed set $\mathcal{D}=\left\{\left(S, E, I_{a}, I_{s}, H, R\right) \in \mathbb{R}_{+}^{6} ; N \leq \frac{\pi}{\mu}\right\}$, is positively invariant and attracts all positive solutions of the model.

Proof Consider the positive domain $\mathcal{D}=\left\{\left(S, E, I_{a}, I_{s}, H, R\right) \in \mathbb{R}_{+}^{6} ; N \leq \frac{\pi}{\mu}\right\}$, the rate of change of the total population is given by;

$$
\frac{d N}{d t}=\pi-\mu(S+R)-\sigma\left(I_{s}+h H\right)
$$

We observe that $N>\frac{\pi}{\mu}$ whenever $\frac{d N}{d t}<0$ and since the right hand side of $\frac{d N}{d t}$ is always bounded, and by standard comparison Theorem in [2], it can be shown that $N(t) \leq N(0) e^{-\mu t}+\frac{\pi}{\mu}\left(1-e^{-\mu t}\right)$. Therefore $\lim _{t \rightarrow \infty} \sup N(t)=\frac{\pi}{\mu}$, implying that if $N(0) \leq \frac{\pi}{\mu}$ then $N(t) \leq \frac{\pi}{\mu}$. The domain $\mathcal{D}$ is positively invariant under the flow of system (1). Therefore, system (1) is biologically feasible and mathematically well posed in $\mathcal{D}$. 
3.2 Local stability of the disease free equilibrium and computation of $\mathcal{R}_{0}$

The model system $(1)$ has a disease free equilibrium $\left(\xi_{0}\right)$ given by $\xi_{0}=(\pi / \mu, 0,0,0,0,0)$. The local stability of $\xi_{0}$, is determined using the next generation matrix described in Driessche and Watmough [1]. The basic reproduction number, $\mathcal{R}_{0}$ (the average number of secondary infections generated by a typical COVID-19 asymptomatic and symptomatic infectious individual during their entire infectious period) used to understand the disease dynamics and ascertain the effectiveness of implemented control strategies is also obtained by the same method. We set $m=1$ due to the assumption that there is no reduction in transmission from asymptomatic individuals. Therefore, we obtain the derivatives of the matrix expressions for the new infections and transition states. These are evaluated at the $\xi_{0}$ and are given by,

$$
F=\left(\begin{array}{cccc}
0 & \frac{b \beta \pi}{A \mu} & \frac{b \beta \pi}{A \mu} & \frac{b \beta g \pi}{A \mu} \\
0 & 0 & 0 & 0 \\
0 & 0 & 0 & 0 \\
0 & 0 & 0 & 0
\end{array}\right), \quad V=\left(\begin{array}{cccc}
\rho & 0 & 0 & 0 \\
-\rho r & \omega_{a} & 0 & 0 \\
-\rho k_{1} & 0 & \omega_{s}+\sigma & 0 \\
-c \rho & -\omega_{a} & -\omega_{s} & \alpha+\sigma h
\end{array}\right)
$$

with

$$
F V^{-1}=\left(\begin{array}{cccc}
\frac{\beta G \pi}{A \mu} & \frac{b \beta \pi\left(\alpha+g \omega_{a}+\sigma h\right)}{A \mu \omega_{a}(\alpha+\sigma h)} & \frac{b \beta \pi\left(\alpha+g \omega_{s}+\sigma h\right)}{A \mu\left(\omega_{s}+\sigma\right)(\alpha+\sigma h)} & \frac{b \beta g \pi}{A \mu(\alpha+\sigma h)} \\
0 & 0 & 0 & 0 \\
0 & 0 & 0 & 0 \\
0 & 0 & 0 & 0
\end{array}\right)
$$

where

$$
\begin{aligned}
G & =b k_{1} \omega_{a}(\alpha+h \sigma)+b g\left(\sigma(c+r)+\omega_{s}\right)+b r(\alpha+h \sigma)\left(\omega_{s}+\sigma\right), \\
k_{1} & =1-c-r, \quad k_{2}=1-a-e, \quad k_{3}=1-a, \quad k_{4}=1-r, \quad k_{5}=1-c .
\end{aligned}
$$

The spectral radius, $\rho$, of matrix $F V^{-1}$ gives the reproduction number as,

$$
\mathcal{R}_{0}=\rho\left(F V^{-1}\right)=\mathcal{R}_{I_{a}}+\mathcal{R}_{I_{s}}+\mathcal{R}_{h}
$$

where $\mathcal{R}_{I_{a}}=\frac{\pi b \beta r}{A \mu \omega_{a}}, \quad \mathcal{R}_{I_{s}}=\frac{\pi b \beta k_{1}}{A \mu\left(\omega_{s}+\sigma\right)}, \quad \mathcal{R}_{h}=\frac{\pi b \beta g\left(\sigma(c+r)+\omega_{s}\right)}{A \mu(\alpha+h \sigma)\left(\omega_{s}+\sigma\right)}$.

By Theorem 2 of [1], the following lemma is established.

Lemma 1 The DFE, $\xi_{0}$, of the model is locally asymptotically stable if $\mathcal{R}_{0}<1$ and unstable if $\mathcal{R}_{0}>1$.

Lemma 1 epidemiologically implies that a slight influx in COVID-19 cases can not result into an outbreak. The COVID-19 threshold $\mathcal{R}_{0}$ estimated under the assumption of population density dependent contact rate, constitutes the reproduction numbers associated with the asymptomatically infected $\mathcal{R}_{I_{a}}$, the symptomatic $\mathcal{R}_{I_{s}}$ and the hospitalised $\mathcal{R}_{h}$ individuals. All these three expressions highlight the significance of the infectious period $1 / \omega_{a}$ and $\left(1 /\left(\omega_{s}+\sigma\right)\right)$, susceptible fraction $(b)$ and habitat area size $(A)$ on the magnitude of $\mathcal{R}_{0}$. For example, in relation to COVID-19 dynamics, shortening the infectious period can be achieved through improved contact tracing and surveillance. The susceptible fraction can be reduced by lock-downs and the use of masks. Since the habitat size translates directly into population density, the effect of area size can be implicitly explored by reducing the total population or increasing the size of the area occupied. These effects are further explored numerically as exhibited in Section 4.

In a situation with no fatalities, $\mathcal{R}_{I_{a}}$ and $\mathcal{R}_{I_{s}}$ are inversely proportional to the habitat area size and the efforts employed in tracing and hospitalising asymptomatically and symptomatically infected individuals. This implies that if the surveillance and contact tracing efforts are not sufficient to ensure timely identification and hospitalisation of infectious individuals, then the number of new cases caused by each infectious individual would be more than double the threshold of one.

On the impact of area occupancy on the number of new cases, it is observed that $\mathcal{R}_{0}$ is inversely proportional to the area. This implies that the smaller the area, the bigger the number of expected new cases. The critical area, $\left(A^{*}\right)$ below which the disease dies out is determined to analyse the relationship that exists between the area and effective contact tracing. This is obtained by equating $\mathcal{R}_{0}$ to one such that,

$$
A^{*}\left(\omega_{a}, \omega_{s}\right)=\frac{\pi b \beta r}{\mu \omega_{a}}+\frac{\pi b \beta k_{1}}{\mu\left(\omega_{s}+\sigma\right)}+\frac{\pi b \beta g\left(\sigma(c+r)+\omega_{s}\right)}{\mu(\alpha+h \sigma)\left(\omega_{s}+\sigma\right)} .
$$

From expression (2), we notice that critical area is directly proportional to time taken to identify and hospitalise infected individuals. This continues to emphasise the importance of effective contact tracing in crowded and high activity areas if COVID-19 is to be mitigated. 
On social distancing and proper use of masks to manage COVID-19, $\mathcal{R}_{0}$ is directly proportional to the proportion of susceptible individuals $(b)$. It is clear that with proper use of masks and proper exercising of social distancing practice, then the number of new cases will be minimized and this will reduce on the burden of the disease and eventually die out.

On the impact of hospital acquired infections, the limit as $g$ tends to zero and $\mathcal{R}_{0}$ can be obtained from the sum of $\mathcal{R}_{I_{a}}$ and $\mathcal{R}_{I_{s}}$.

3.3 Global stability of disease free equilibrium

Lemma 2 The DFE, $\xi_{0}$, of the model is globally asymptotically stable in the invariant region $\mathcal{D}$ if $\mathcal{R}_{0}<1$ and unstable if $\mathcal{R}_{0}>1$.

Proof Consider the positively definite Lyapunov function,

$$
V=(\alpha+\sigma h) E+\frac{\pi b \beta\left(g \omega_{a}+\alpha+h \sigma\right)}{A \mu \omega_{a}} I_{a}+\frac{\pi b \beta\left(\alpha+g \omega_{s}+h \sigma\right)}{A \mu\left(\omega_{s}+\sigma\right)} I_{s}+\frac{\pi b \beta g}{A \mu} H .
$$

The time-derivative of function (3) is given by,

$$
\begin{aligned}
\frac{d V}{d t}= & (\alpha+\sigma h) \frac{d E}{d t}+\left(\frac{b \beta \pi\left(\alpha+g \omega_{a}+\sigma h\right)}{A \mu \omega_{a}}\right) \frac{d I_{a}}{d t}+\left(\frac{b \beta \pi\left(\alpha+g \omega_{s}+\sigma h\right)}{A \mu\left(\sigma+\omega_{s}\right)}\right) \frac{d I_{s}}{d t}+\left(\frac{g \beta b \pi}{A \mu}\right) \frac{d H}{d t}, \\
= & (\alpha+\sigma h)\left(\frac{b \beta p S\left(g H+I_{a}+I_{s}\right)}{A}+e \pi-\rho E\right)+\left(\frac{b \beta \pi\left(\alpha+g \omega_{a}+\sigma h\right)}{A \mu \omega_{a}}\right) \\
& \left(a \pi-\omega_{a} I_{a}+r \rho E\right)+\left(\frac{b \beta \pi\left(\alpha+g \omega_{s}+\sigma t\right)}{A \mu\left(\sigma+\omega_{s}\right)}\right)\left(\rho k_{1} E-\sigma I_{s}-\omega_{s} I_{s}\right) \\
& +\left(\frac{g \beta b \pi}{A \mu}\right)\left(c \rho E-\alpha H-\sigma h H+\omega_{a} I_{a}+\omega_{s} I_{s}\right), \\
\leq & (\alpha+\sigma h)\left(\frac{b \beta p \pi\left(g H+I_{a}+I_{s}\right)}{A \mu}+e \pi-\rho E\right)+\left(\frac{b \beta \pi\left(\alpha+g \omega_{a}+\sigma h\right)}{A \mu \omega_{a}}\right) \\
& \left(a \pi-\omega_{a} I_{a}+r \rho E\right)+\left(\frac{b \beta \pi\left(\alpha+g \omega_{s}+\sigma t\right)}{A \mu\left(\sigma+\omega_{s}\right)}\right)\left(\rho k_{1} E-\sigma I_{s}-\omega_{s} I_{s}\right) \\
& +\left(\frac{g \beta b \pi}{A \mu}\right)\left(c \rho E-\alpha H-\sigma h H+\omega_{a} I_{a}+\omega_{s} I_{s}\right), \\
\leq & \pi\left(\frac{a b \beta \pi\left(\alpha+g \omega_{a}+\sigma h\right)}{A \mu \omega_{a}}+e(\alpha+\sigma h)\right)+\rho E(\alpha+h \sigma)\left(\mathcal{R}_{0}-1\right), \\
\leq & \pi\left(\frac{a b \beta \pi\left(\alpha+g \omega_{a}+\sigma h\right)}{A \mu \omega_{a}}+e(\alpha+\sigma h)\right)+\rho E(\alpha+h \sigma)\left(\mathcal{R}_{0}-1\right), \\
\frac{d V}{d t} \leq & \left.-\rho E(\alpha+h \sigma)(1-\mathcal{R})_{0}\right), \text { since } a=0 \text { and } e=0 \text { at the disease free equilibrium } \xi_{0} .
\end{aligned}
$$

When $\mathcal{R}_{0} \leq 1, \frac{d V}{d t}$ is negative semi-definite, hence the largest compact invariant set in $\mathcal{D}$ such that $\frac{d V}{d t}=0$ when $\mathcal{R}_{0} \leq 1$ is the singleton $\xi_{0}$. By the LaSalle Invariance Principle [3], we conclude that the disease free equilibrium $\xi_{0}$ is globally asymptotically stable in $\mathcal{D}$ if $\mathcal{R}_{0} \leq 1$ and unstable otherwise.

3.4 Stability analysis of the unique endemic equilibrium point without infected recruitments

Let $\xi^{*}=\left\{S^{*}, E^{*}, I_{a}^{*}, I_{s}^{*}, H^{*}, R^{*}\right\}$ be the generic equilibrium point and $\lambda=\left(\beta b\left(I_{a}+I_{s}+g H\right)\right) / A$ be the force of infection, then equilibrium points are obtained in terms of the force of infection at steady state denoted as $\left(\lambda^{*}\right)$ which must satisfy the quadratic equation

$$
A_{3} \lambda^{* 2}+A_{2} \lambda^{*}+A_{1}=0
$$

where

$$
\begin{aligned}
A_{1}= & -\pi b \beta \mu(\mu+\tau)\left(\omega_{a}\left(g(a+e) \omega_{s}+a g \sigma+e \sigma\left(g(c+r)+h k_{1}\right)+\alpha e k_{1}\right)+(a+e r)(\alpha+h \sigma)\left(\omega_{s}+\sigma\right)\right) \\
A_{2}= & A \mu \omega_{a}(\mu+\tau)(\alpha+h \sigma)\left(\omega_{s}+\sigma\right)+\pi b \beta\left(\sigma \omega_{a}(\mu+\tau)\left((h-g)\left(a k_{1}+c+r\right)-h\right)\right. \\
& \left.-g \omega_{a}(\mu+\tau) \omega_{s}-\left(\omega_{s}+\sigma\right)\left(a k_{4}(\alpha \mu+h \sigma(\mu+\tau))+r(\mu+\tau)(\alpha+h \sigma)\right)-\alpha k_{1} \omega_{a}\left(k_{3} \mu+\tau\right)-a \alpha k_{1} \sigma \tau\right) \\
A_{3}= & A \omega_{a}\left(\sigma\left(h \sigma(\mu+\tau)+\alpha\left(k_{1} \tau+\mu\right)\right)+\omega_{s}(\alpha \mu+h \sigma(\mu+\tau))\right) .
\end{aligned}
$$


Then the equilibrium points are obtained by solving equation (4) and substituting the positive values of $\lambda^{*}$ into the equilibrium point $\xi^{*}$.

Without infected arrivals, then the parameters $a$ and $e$ (the proportions of asymptomatically and latently infected individuals respectively recruited into a closed community) are set to zero to obtain,

$$
A_{3} \lambda^{* 2}+A_{2} \lambda^{*}=0
$$

Clearly, $\lambda^{*}=0$ is a solution and in this case, corresponding to $\xi_{0}$ and when $\lambda^{*} \neq 0$, the coefficient $A_{2}$ summarizes as

$$
A_{2}=A \mu \omega_{a}(\mu+\tau)(\alpha+h \sigma)\left(\omega_{s}+\sigma\right)\left(1-\mathcal{R}_{0}\right)
$$

Since $A_{3}$ is always positive, then if $\mathcal{R}_{0}>1$, we have $A_{3} \lambda^{*}+A_{2}=0$ implying that $\lambda^{*}=\frac{-A_{2}}{A_{3}}$. In this case, a unique endemic equilibrium point $\left(\xi_{1}\right)$ is obtained as;

$$
\begin{aligned}
S_{1} & =\frac{\pi(\mu+\tau)(\alpha+h \sigma)\left(\omega_{s}+\sigma\right)}{\left(\omega_{s}+\sigma\right)\left(\alpha \mu\left(\lambda^{*}+\mu+\tau\right)+h \sigma\left(\lambda^{*}+\mu\right)(\mu+\tau)\right)+\alpha \lambda^{*} k_{1} \sigma \tau} \\
E_{1} & =\frac{\pi \lambda^{*}(\mu+\tau)(\alpha+h \sigma)\left(\omega_{s}+\sigma\right)}{\rho\left(\left(\omega_{s}+\sigma\right)\left(\alpha \mu\left(\lambda^{*}+\mu+\tau\right)+h \sigma\left(\lambda^{*}+\mu\right)(\mu+\tau)\right)+\alpha \lambda^{*} k_{1} \sigma \tau\right)} \\
I_{a_{1}} & =\frac{\pi \lambda^{*} r(\mu+\tau)(\alpha+h \sigma)\left(\omega_{s}+\sigma\right)}{\omega_{a}\left(\left(\omega_{s}+\sigma\right)\left(\alpha \mu\left(\lambda^{*}+\mu+\tau\right)+h \sigma\left(\lambda^{*}+\mu\right)(\mu+\tau)\right)+\alpha \lambda^{*} k_{1} \sigma \tau\right)} \\
I_{s_{1}} & =\frac{\pi \lambda^{*} k_{1}(\mu+\tau)(\alpha+h \sigma)}{\left(\omega_{s}+\sigma\right)\left(\alpha \mu\left(\lambda^{*}+\mu+\tau\right)+h \sigma\left(\lambda^{*}+\mu\right)(\mu+\tau)\right)+\alpha \lambda^{*} k_{1} \sigma \tau} \\
H_{1} & =\frac{\pi \lambda^{*}(\mu+\tau)\left(\sigma(c+r)+\omega_{s}\right)}{\left(\omega_{s}+\sigma\right)\left(\alpha \mu\left(\lambda^{*}+\mu+\tau\right)+h \sigma\left(\lambda^{*}+\mu\right)(\mu+\tau)\right)+\alpha \lambda^{*} k_{1} \sigma \tau} \\
R_{1} & =\frac{\pi \lambda^{*}(\mu+\tau)\left(\sigma(c+r)+\omega_{s}\right)}{\left(\omega_{s}+\sigma\right)\left(\alpha \mu\left(\lambda^{*}+\mu+\tau\right)+h \sigma\left(\lambda^{*}+\mu\right)(\mu+\tau)\right)+\alpha \lambda^{*} k_{1} \sigma \tau}
\end{aligned}
$$

The local stability of the endemic equilibrium $\xi_{1}$ is obtained using the Center Manifold Theorem 4.1 in [4] and the following result is established

Lemma 3 The unique endemic equilibrium point $\xi_{1}$ is unstable when $\mathcal{R}_{0}>1$.

Proof Lets redefine the state variable $\left(S, E, I_{a}, I_{s}, H, R\right)$ as $\left(x_{1}, x_{2}, x_{3}, x_{4}, x_{5}, x_{6}\right)$ then the associated system $(1)$ is given as;

$$
\begin{aligned}
\frac{d x_{1}}{d t} & =f_{1}=(1-(e+a)) \pi-\frac{\beta b x_{1}\left(x_{3}+x_{4}+g x_{5}\right)}{A}-\mu x_{1}+\tau x_{6}, \\
\frac{d x_{2}}{d t} & =f_{2}=e \pi+\frac{\beta b x_{1}\left(x_{3}+x_{4}+g x_{5}\right)}{A}-\rho x_{2}, \\
\frac{d x_{3}}{d t} & =f_{3}=a \pi+r \rho x_{2}-\omega_{a} x_{3}, \\
\frac{d x_{4}}{d t} & =f_{4}=(1-(c+r)) \rho x_{2}-\sigma x_{4}-\omega_{s} x_{4}, \\
\frac{d x_{5}}{d t} & =f_{5}=c \rho x_{2}+\omega_{a} x_{3}+\omega_{s} x_{4}-h \sigma x_{5}-\alpha x_{5}, \\
\frac{d x_{6}}{d t} & =f_{6}=\alpha x_{5}-\mu x_{6}-\tau x_{6} .
\end{aligned}
$$

The bifurcation parameter $\phi$ obtained by equating $\mathcal{R}_{0}$ to one is given by

$$
\phi=\beta^{*}=\frac{A \mu \omega_{a}(\alpha+h \sigma)\left(\omega_{s}+\sigma\right)}{\pi b\left(\alpha k_{1} \omega_{a}+\sigma((c+r)(g-h)+h)+g \omega_{s}+r(\alpha+h \sigma)\left(\omega_{s}+\sigma\right)\right)}
$$

By Linearizing system 5 at disease free equilibrium $\left(\xi_{0}\right)$ and with $\phi$ the bifurcation parameter, we obtain

$$
J_{\xi_{0}}=\left(\begin{array}{cccccc}
-\mu & 0 & -\frac{b \phi \pi}{A \mu} & -\frac{b \phi \pi}{A \mu} & -\frac{b \phi g \pi}{A \mu} & \tau \\
0 & -\rho & \frac{b \phi \pi}{A \mu} & \frac{b \phi \pi}{A \mu} & \frac{b \phi g \pi}{A \mu} & 0 \\
0 & \rho r & -\omega_{a} & 0 & 0 & 0 \\
0 & \rho k_{1} & 0 & -\sigma-\omega_{s} & 0 & 0 \\
0 & 0 & \omega_{a} & \omega_{s} & -h \sigma & -\alpha \\
0 & 0 & 0 & 0 & \alpha & -\mu-\tau
\end{array}\right)
$$


The Jacobian matrix $J_{\xi_{0}}$ has zero eigenvalue and the rest are negative. The left eigenvector associated with the zero eigenvalue of $(6)$ is given by $v=\left(v_{1}, v_{2}, v_{3}, v_{4}, v_{6}\right)^{T}$ where,

$$
\begin{array}{ll}
v_{1}=0, \quad v_{2}=-\frac{A \mu v_{6}\left(\alpha^{2}+\sigma h(\mu+\tau)\right)}{\alpha b \beta^{*} g \pi}, & v_{3}=-\frac{v_{6}\left(\alpha^{2}+g \omega_{a}(\mu+\tau)+h \sigma(\mu+\tau)\right)}{\alpha g \omega_{a}}, \\
v_{4}=-\frac{v_{6}\left(\alpha^{2}+g(\mu+\tau) \omega_{s}+h \sigma(\mu+\tau)\right)}{\alpha g\left(\omega_{s}+\sigma\right)}, & v_{5}=-\frac{v_{6}(\mu+\tau)}{\alpha},
\end{array}
$$

Similarly, the right eigenvector of (6) associated with the zero eigenvalue is given by $w=\left(w_{1}, w_{2}, w_{3}, w_{4}, w_{6}\right)^{T}$ with $v . w=1$ where,

$$
\begin{aligned}
& w_{1}=\frac{-\frac{\pi b \beta^{*} r\left(\omega_{s}+\sigma\right)\left(\frac{h \sigma w_{6}(\mu+\tau)}{\alpha}+\alpha w_{6}\right)}{\omega_{a}\left((1-c) \omega_{s}+r \sigma\right)}+A \mu \tau w_{6}-\frac{\pi b \beta^{*} k_{1}\left(\frac{h \sigma w_{6}(\mu+\tau)}{\alpha}+\alpha w_{6}\right)}{(1-c) \omega_{s}+r \sigma}-\frac{\pi b \beta^{*} g w_{6}(\mu+\tau)}{\alpha}}{A \mu^{2}}, \\
& w_{2}=\frac{w_{6}\left(\omega_{s}+\sigma\right)\left(\alpha^{2}+h \sigma(\mu+\tau)\right)}{\alpha \rho\left((1-c) \omega_{s}+r \sigma\right)}, \quad w_{3}=\frac{r\left(\omega_{s}+\sigma\right)\left(\frac{h \sigma w 6(\mu+\tau)}{\alpha}+\alpha w_{6}\right)}{\omega_{a}\left((1-c) \omega_{s}+r \sigma\right)}, \\
& w_{4}=\frac{k_{1}\left(\frac{h \sigma w_{6}(\mu+\tau)}{\alpha}+\alpha w_{6}\right)}{(1-c) \omega_{s}+r \sigma}, \quad w_{5}=\frac{w_{6}(\mu+\tau)}{\alpha}, \quad w_{6}>0 .
\end{aligned}
$$

Next we compute the non-zero partial derivatives of system (6) with respect to the state variables that are used in the computation of coefficients $\mathbf{a}$ and $\mathbf{b}$ defined as,

$$
\begin{aligned}
& \mathbf{a}=\sum_{i, j, k=1}^{n} v_{k} w_{i}, w_{j} \frac{\partial^{2} f_{k}}{\partial x_{i} \partial x_{j}}(0,0) \\
& \mathbf{b}=\sum_{i, k=1}^{n} v_{k} w_{i} \frac{\partial^{2} f_{k}}{\partial x_{i} \partial \phi}(0,0) .
\end{aligned}
$$

Thus, we obtain,

$$
\begin{aligned}
& \frac{\partial^{2} f_{1}}{\partial x_{1} \partial x_{3}}=-\frac{b \beta^{*}}{A}, \quad \frac{\partial^{2} f_{1}}{\partial x_{1} \partial x_{4}}=-\frac{b \beta^{*}}{A}, \quad \frac{\partial^{2} f_{1}}{\partial x_{1} \partial x_{5}}=-\frac{b \beta^{*} g}{A}, \quad \frac{\partial^{2} f_{1}}{\partial x_{3} \partial x_{1}}=-\frac{b \beta^{*}}{A}, \\
& \frac{\partial^{2} f_{1}}{\partial x_{4} \partial x_{1}}=-\frac{b \beta^{*}}{A}, \quad \frac{\partial^{2} f_{1}}{\partial x_{5} \partial x_{1}}=-\frac{b \beta^{*} g}{A} \frac{\partial^{2} f_{2}}{\partial x_{1} \partial x_{3}}=\frac{b \beta^{*}}{A}, \quad \frac{\partial^{2} f_{2}}{\partial x_{1} \partial x_{4}}=\frac{b \beta^{*}}{A}, \\
& \frac{\partial^{2} f_{2}}{\partial x_{1} \partial x_{5}}=\frac{b \beta^{*} g}{A}, \quad \frac{\partial^{2} f_{2}}{\partial x_{3} \partial x_{1}}=\frac{b \beta^{*}}{A}, \quad \frac{\partial^{2} f_{2}}{\partial x_{4} \partial x_{1}}=\frac{b \beta^{*}}{A}, \quad \frac{\partial^{2} f_{2}}{\partial x_{5} \partial x_{1}}=-\frac{b \beta^{*} g}{A} .
\end{aligned}
$$

Following Theorem 4.1 in [4], the non-zero partial derivatives of system (5) with respect to state variables and the bifurcation parameter $\phi$ that are give by;

$$
\begin{aligned}
\frac{\partial^{2} f_{1}}{\partial x_{3} \partial \phi}=-\frac{b \pi}{A \mu}, \quad \frac{\partial^{2} f_{1}}{\partial x_{4} \partial \phi}=-\frac{b \pi}{A \mu}, \quad \frac{\partial^{2} f_{1}}{\partial x_{5} \partial \phi}=-\frac{b \pi g}{A \mu}, \\
\frac{\partial^{2} f_{2}}{\partial x_{3} \partial \phi}=\frac{b \pi}{A \mu}, \quad \frac{\partial^{2} f_{2}}{\partial x_{4} \partial \phi}=\frac{b \pi}{A \mu} \frac{\partial^{2} f_{2}}{\partial x_{5} \partial \phi}=\frac{b \pi g}{A \mu} .
\end{aligned}
$$

By substituting the $v, w$, and expressions above into (7) we obtain,

$$
\begin{aligned}
\mathbf{a} & =-\frac{2 b \beta^{*}\left(w_{3}+w_{4}+g w_{5}\right)\left(v_{1} w_{1}-v_{2} w_{2}\right)}{A} . \quad \text { Since } v_{1}=0, \text { it simplifies to, } \\
& =\frac{2 b \beta^{*}\left(w_{3}+w_{4}+g w_{5}\right) v_{2} w_{2}}{A}, \quad \text { which is negative because } v_{2}<0 . \\
\mathbf{b} & =\frac{b \pi v_{2}\left(w_{3}+w_{4}+g w_{5}\right)}{A \mu} . \quad \text { Since } v_{2}<0, \text { then } \mathbf{b}<0 .
\end{aligned}
$$

Thus, according to condition (ii) of Theorem 4.1 in [4], it can be concluded that the model system (1) with $a=0$ and $e=0$, has an unstable endemic equilibrium point $\xi_{1}$. 
The other endemic equilibrium points that persist in the community when infected individuals are recruited into the community are obtained by investigating the nature of the coefficients of the polynomial (4). When $a \neq 0$ and $e \neq 0, A_{3}$ is positive, $A_{1}$ is negative, then we have one positive root of (4) irrespective of the sign of the coefficient $A_{2}$. Thus the endemic equilibrium point $\xi_{2}$ is given by;

$$
\begin{aligned}
S_{2}= & \frac{\pi\left(\alpha \tau\left(\sigma\left(1-e k_{1}\right)+\omega_{s}\right)+k_{2}\left(\omega_{s}+\sigma\right)(\alpha \mu+h \sigma(\mu+\tau))\right)}{\left(\omega_{s}+\sigma\right)\left(\alpha \mu\left(\lambda^{*}+\mu+\tau\right)+h \sigma\left(\lambda^{*}+\mu\right)(\mu+\tau)\right)+\alpha \lambda^{*} k_{1} \sigma \tau}, \\
E_{2}= & \frac{\pi\left(\omega_{s}+\sigma\right)\left(\alpha \lambda^{*} \tau+e \mu(\mu+\tau)(\alpha+h \sigma)+\lambda^{*} k_{3}(\alpha \mu+h \sigma(\mu+\tau))\right)}{\rho\left(\left(\omega_{s}+\sigma\right)\left(\alpha \mu\left(\lambda^{*}+\mu+\tau\right)+h \sigma\left(\lambda^{*}+\mu\right)(\mu+\tau)\right)+\alpha \lambda^{*} k_{1} \sigma \tau\right)}, \\
I_{a_{2}}= & \frac{\pi\left(\left(\omega_{s}+\sigma\right)\left((\mu+\tau)(\alpha+h \sigma)\left(a \mu+r\left(e \mu+\lambda^{*}\right)\right)+a \lambda^{*} k_{4}(\alpha \mu+h \sigma(\mu+\tau))\right)+a \alpha \lambda^{*} k_{1} \sigma \tau\right)}{\omega_{a}\left(\left(\omega_{s}+\sigma\right)\left(\alpha \mu\left(\lambda^{*}+\mu+\tau\right)+h \sigma\left(\lambda^{*}+\mu\right)(\mu+\tau)\right)+\alpha \lambda^{*} k_{1} \sigma \tau\right)}, \\
I_{s_{2}}= & \frac{\pi k_{1}\left(\alpha \lambda^{*} \tau+e \mu(\mu+\tau)(\alpha+h \sigma)+\lambda^{*} k_{3}(\alpha \mu+h \sigma(\mu+\tau))\right)}{\left(\omega_{s}+\sigma\right)\left(\alpha \mu\left(\lambda^{*}+\mu+\tau\right)+h \sigma\left(\lambda^{*}+\mu\right)(\mu+\tau)\right)+\alpha \lambda^{*} k_{1} \sigma \tau}, \\
H_{2}= & \frac{\pi(\mu+\tau)\left(\sigma\left(a \mu+(c+r)\left(e \mu+\lambda^{*}\right)\right)+\omega_{s}\left(\mu(a+e)+\lambda^{*}\right)+a \lambda^{*} k_{1} \sigma\right)}{\left(\omega_{s}+\sigma\right)\left(\alpha \mu\left(\lambda^{*}+\mu+\tau\right)+h \sigma\left(\lambda^{*}+\mu\right)(\mu+\tau)\right)+\alpha \lambda^{*} k_{1} \sigma \tau}, \\
R_{2}= & \frac{\pi \alpha\left(\sigma\left(a \mu+(c+r)\left(e \mu+\lambda^{*}\right)\right)+\omega_{s}\left(\mu(a+e)+\lambda^{*}\right)+a \lambda^{*} k_{1} \sigma\right)}{\left(\omega_{s}+\sigma\right)\left(\alpha \mu\left(\lambda^{*}+\mu+\tau\right)+h \sigma\left(\lambda^{*}+\mu\right)(\mu+\tau)\right)+\alpha \lambda^{*} k_{1} \sigma \tau},
\end{aligned}
$$

The results obtained above show that the disease will always persist as long as infected individual are allowed into the community. It is important to note that all the endemic equilibrium points have the basic reproduction number inbuilt in them implying that all control measures targeting the reduction of $\mathcal{R}_{0}$ will directly affect the size of these equilibrium points. From the computation of $\mathcal{R}_{0}$, the number of new cases in a given community is inversely proportional to the area. This indicates that the size of the equilibrium points will also be inversely proportion to the area implying that with a small area, if $\mathcal{R}_{0}$ is even slightly greater than one then there will be an outbreak. Furthermore, equilibrium point $\xi_{2}$ will always exist irrespective of the value $\mathcal{R}_{0}$. This implies that control measures such as social distancing and reducing on the congestion would be the only feasible approaches to manage the disease in a community with such settings.

\section{Numerical simulation}

In this Section, numerical simulations are presented to gain more insights on the model properties under various scenarios. Simulations are performed using Mathematica ®12.0 (Wolfram Research, Inc.) software. The parameters values given in Table 1 are obtained from literature and some are assumed.

\subsection{Impact of SOPs on the basic reproduction number, $\mathcal{R}_{0}$}

In this section, we investigate the impact of SOPs on the value of the basic reproduction number. All simulation results in Fig. 1 show that as the level of adherence to SOPs increases, basic reproduction number will decrease. In Fig. 1(a), it is observed that $\mathcal{R}_{0}<1$ over the entire explored area allocation per individual if only $10 \%$ of the population did not observe (SOPs). However, if $50 \%$ or $90 \%$ are unprotected, $\mathcal{R}_{0}<1$ only if we ensure area allocations of $0.00081 \mathrm{~km}^{2}$ and $0.0015 \mathrm{~km}^{2}$ per individual respectively (i.e., population densities of 1,230 and 675 individuals per $\mathrm{km}^{2}$ ). These findings support adoption of measures to reduce crowding in existing refugee settlements e.g., relocation of people to less crowded settlements, as well as limiting new arrivals in the already crowded settlements. These results also echo the importance of proper adherence to the existing control measures that limit susceptibility.

From Fig. 1(b), it can be seen that $\mathcal{R}_{0}<1$ for all explored infectivity levels of the hospitalised, if nonadherence to SOPs is kept at $10 \%$ and at $50 \%$, infectivity levels of the hospitalised need to be at $<2.5 \%$ to have $\mathcal{R}_{0}<1$. On the other hand, if non-adherence is at $90 \%$, then even without hospital-acquired infections, $\mathcal{R}_{0}>1$. Results show that, in settlements with a population density of 1,080 individuals per $\mathrm{km}^{2}$, ensuring $50 \%$ mingling and susceptibility may not be sufficient to avert the epidemic if the hospitalised are $>2.5 \%$ infectious (compared to the free-living infected). Thus, elimination of hospital acquired infections is key to preventing an epidemic situation even in partial locked-down populations.

Effective surveillance and contact tracing is key in reducing the infectious period of a COVID-19 asymptomatic patient who may remain unidentified in the community for an extended period, or the symptomatic 
Table 1 Table showing parameter values used in the numerical simulations

\begin{tabular}{|c|c|c|c|}
\hline $\begin{array}{c}\text { Parameter/ } \\
\text { variable }\end{array}$ & Description & Value (units) & Source \\
\hline$N$ & Population in high activity area & 270,000 & {$[23]$} \\
\hline$A$ & Settlement area size & $250\left(\mathrm{~km}^{2}\right)$ & {$[22]$} \\
\hline$a$ & Fraction of arrivals that are asymptomatic & 0.01 & asuumed \\
\hline$e$ & Fraction of arrivals that are latently infected & 0.01 & assumed \\
\hline$\beta$ & Transmission coefficient & $0.00056\left(\mathrm{~km}^{2}\right.$ per day $)$ & {$[21]$} \\
\hline$b$ & Fraction of susceptible individuals that available & 0.1 & assumed \\
\hline$\mu$ & Per capita exit rate of susceptible and recovered individuals from the community & $6.2 \times 10^{-7}$ (per day) & {$[23]$} \\
\hline$\tau$ & Waning rate of immunity & 0 (per day) & assumed \\
\hline$g$ & Infectivity reduction factor among hospitalised $(\in[0,1])$ & 0.005 & assumed \\
\hline$\omega_{a}$ & Hospitalisation rate of asymptomatic infectious & 0.2 (per day) & {$[19-21]$} \\
\hline$\omega_{s}$ & Hospitalisation rate of symptomatic infectious & 0.5 (per day) & {$[19-21]$} \\
\hline$c$ & Fraction of latently infected hospitalised $(\in[0,1])$ & 0.2 & {$[19]$} \\
\hline$r$ & Fraction of latently infected that become asymptomatic $(\in[0,1])$ & 0.4 & {$[19]$} \\
\hline$h$ & Disease induced mortality reduction among hospitalised $(\in[0,1])$ & 0 & {$[19]$} \\
\hline$\rho$ & Progression rate from latent stage to infectious stage & 0.2 (per day) & {$[20]$} \\
\hline$\sigma$ & Disease induced death rate & 0 (per day) & {$[19]$} \\
\hline$\alpha$ & Recovery rate & 0.048 (per day) & {$[19]$} \\
\hline
\end{tabular}

Set to zero since death rate was zero in Uganda as of $20^{\text {th }}$ July 2020.

Set to one pending further studies.

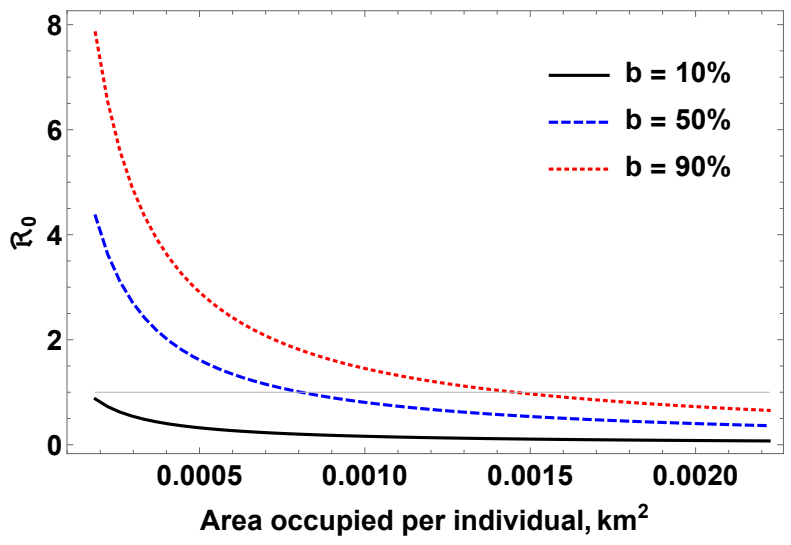

(a)

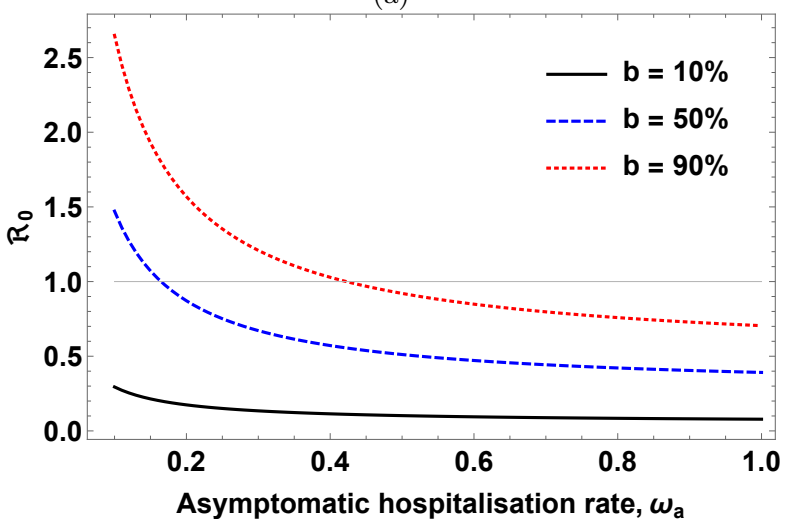

(c)

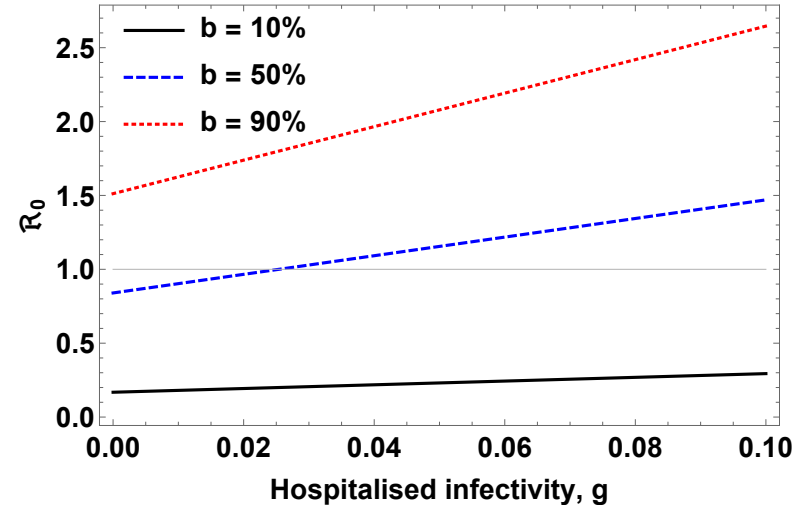

(b)

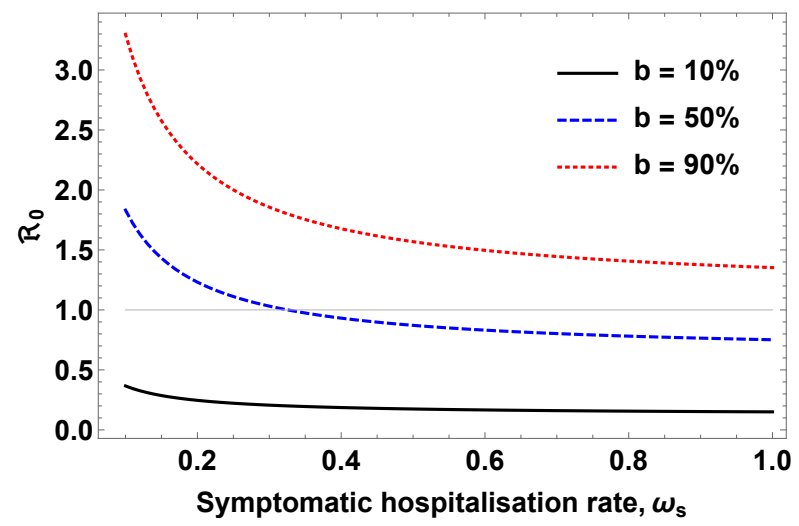

(d)

Fig. 1 Effect of (b) population density, (b) infectivity of hospitalised individuals, (c) hospitalisation rate of asymptomatic infectious individuals and, (d) hospitalisation rate of symptomatic infectious individuals on the basic reproduction number for COVID-19

individual that is fairly easier to identify and hospitalise. Results on the impact of delaying the identification and subsequent hospitalization of infectious individuals (Fig. 1(c) and Fig. 1(d)), show that if 90\% of the population in a settlement with a density of 1,080 individuals per $\mathrm{km}^{2}$ is freely mingling and susceptible, then all infectious individuals must be identified and hospitalized within two days if an epidemic is to be avoided. This requires intensification of contact tracing efforts and may even involving recruitment and regular reinforcement of outbreak response teams. 


\section{Impact of transmission rates on SOPs and disease prevalence}

In this section, we numerically investigate how the variation of transmission rates would influence the adherence to SOPs. The simulations presented show the change in disease prevalence with time for different transmission rates per square kilometers per day under different levels of adherence to the standard operating procedures (SOPs). The parameter (b) which measures the fraction of available susceptible popuplation indicates the percentage of individuals within a community that is not complying with the public health interventions of observing SOPs at any given time. In Fig. 2 (top left), we observe that a low transmission rate of 0.00056 per
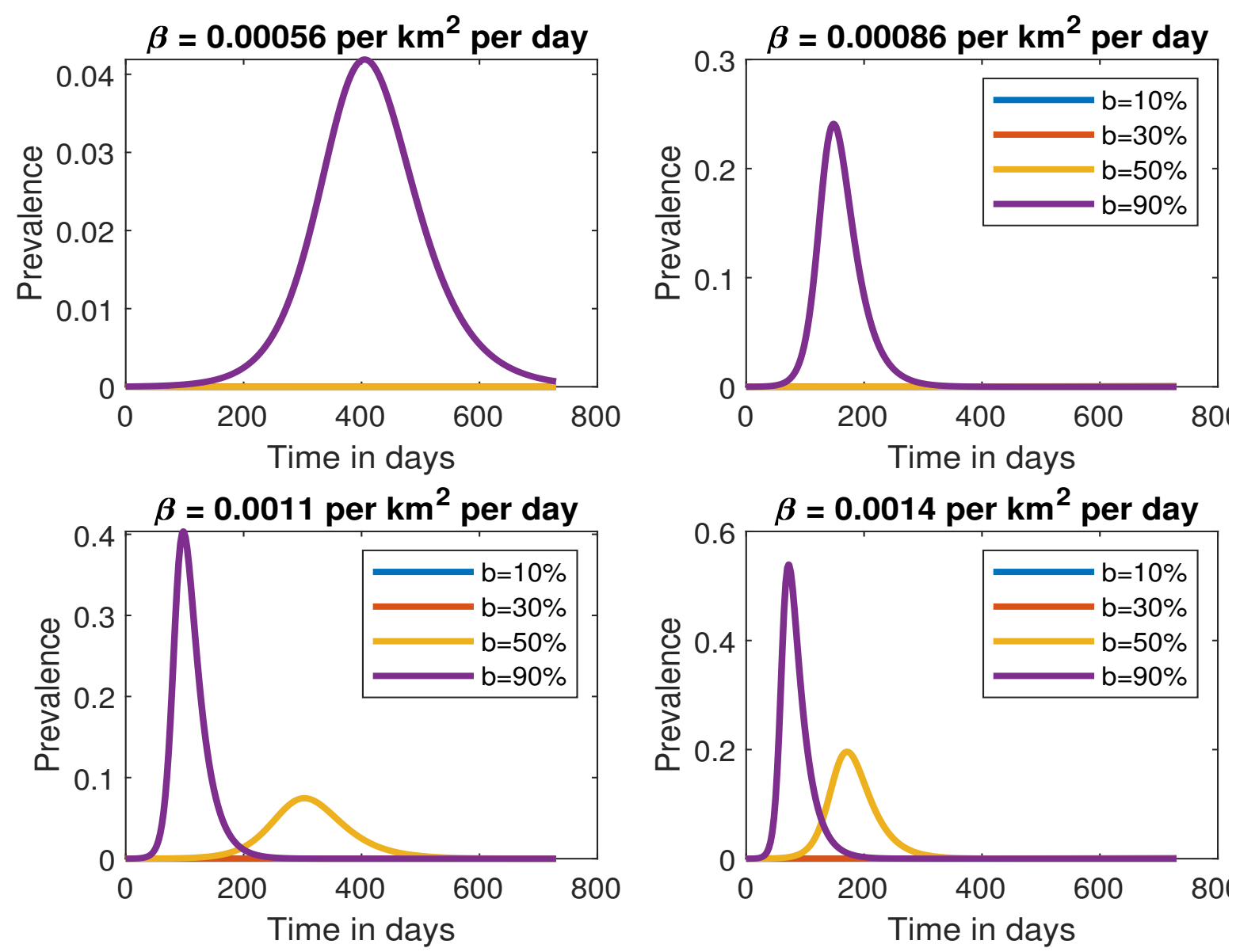

Fig. 2 Estimated COVID-19 prevalence for smaller transmission rate per $\mathrm{km}^{2}$ given different levels of public health interventions

$\mathrm{km}^{2}$ would raise the number of new cases by $4 \%$, attaining the first peak in a period of 400 days when $90 \%$ of the individuals in a highly populated area do not adhere to SOPs. Increasing the transmission rate to 0.00084 per $\mathrm{km}^{2}$ and maintaining the percentage of individuals not adhereing to SOPs at $90 \%$, we notice that the number of new cases rapidly grows to $25 \%$ in a shorter period of only 180 days (Fig. 2, top right). Additionally, when compliance is above $50 \%$, then the peak may never be observed for more than two years. Therefore, keeping the transmission rates low would significantly delay the peaks. This emphasizes that implementation of public health interventions leads to delayed peaks that may eventually lead to the flattening of the curve.

In Fig. 2, bottom plots and Fig. 3, we notice that as the transmission rates increases, then the level of compliance to SOPs needs to increase if the prevalence peaks are to be reduced and delayed. The disease prevalence may peak at $80 \%$ in a period of just 40 days if $90 \%$ of the individuals do not comply with SOPs with a transmission rate of 0.0034 per $\mathrm{km}^{2}$. However, if $70 \%$ (30\% non-adherence) effectively adhere to SOPs, our results show that the peak would be delayed by 60 days with a prevalence of $40 \%$ and as the number of people adhering to SOPs increase to $90 \%$ (10\% non-adherence), then even after a period of two years the peak prevalence will not reached and thus leading to flattening of the epidemic curve. 

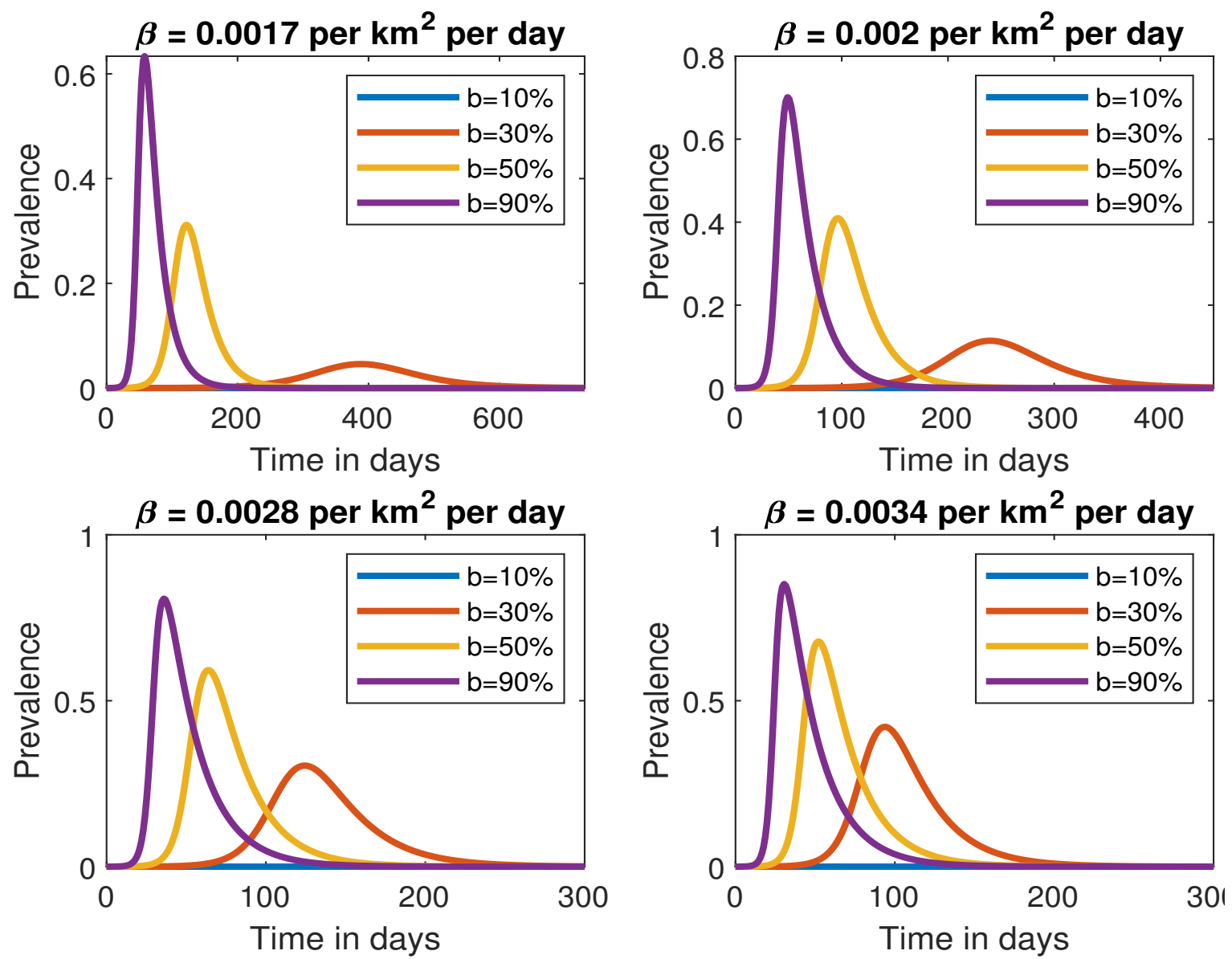

Fig. 3 Estimation of COVID-19 prevalence for slightly higher transmission rates given different levels of adherence to standard operating procedures

\section{Discussion}

The aerosol and possible airborne spread mechanisms of COVID-19 imply that population density is the likely key driving factor in transmission especially in crowded settlements such as refugee camps and slums as well as high activity areas like markets and schools. If properly enforced, physical distancing and closure of high population congregation avenues would help limit disease spread. However, there are challenges in enforcing physical distancing in crowded settlements due to the ways of life in such communities.

Determination of critical population density should be a key component in formulating density-related disease control policies as this would guide feasibility and efficacy assessments of the interventions. Model equilibrium points are obtained and their stability assessed, the disease's density-dependent basic reproduction number is determined and used to calculate the critical population density at which the disease could not be maintained in the settlement. The impact of interventions that: reduce the fraction of susceptible, improve the detection and subsequent hospitalization of infectious individuals and, reduce on the population density in the habitat on COVID-19 transmission dynamics are assessed.

Analytical results show that without entry of infected individuals into the settlement, a globally stable disease free equilibrium exists whenever $\mathcal{R}_{0}<1$ and an unstable endemic equilibrium exists when $\mathcal{R}_{0}>1$. That is, if a single infected person cannot infect at least one susceptible, then the disease dies out naturally without extra effort. The unstable endemic equilibrium implies that if intervention measures such as social distancing and effective use of face masks are well adopted, then COVID-19 new cases can be greatly minimized and the disease could be eradicated. With entry of infected individuals, our analysis show that at any given time, an endemic equilibrium exists and efforts targeting reducing on the number of new cases through effective contact tracing and surveillance may lead to disease eradication.

The analytical expression for $\mathcal{R}_{0}$ highlight the dependence of its magnitude on three major aspects namely, the susceptible fraction, surveillance and contact tracing efforts and population density in the settlement. These factors where hence further assessed quantitatively to determine thresholds for disease spread.

The importance of understanding the disease peak prevalence is to prepare for facilities just in case the disease transmission increases and also be in position to enforce adherence to SOPs if the pandemic curve is 
to be flattened. The results presented in Section 5 provide information on how best to manage COVID-19 in places that are densely populated and the efforts required to enforce SOPs if disease is to be mitigated. Therefore, proper use of masks and physical distancing measures should be enforced in such settings for almost all individuals mingling.

\section{Acknowledgements}

The Authors acknowledge and thank the Government of Uganda and Makerere University Research and Innovation Fund for the grant to carry out this study, and Ministry of Health for the data on COVID-19.

\section{References}

1. van den Driessche P. and Watmough J., Reproduction numbers and sub-threshold endemic equilibria for compartmental models of disease transmission. Math. Biosci., 180:29-48 (2002).

2. Lakshmikantham, S., Leela, S., and Martynyuk, A.A., Stability analysis of nonlinear systems. New York; Basel: Marcel Dekker, Inc (1989).

3. LaSalle, J.P., The stability of dynamical systems. Society for Industrial and Applied Mathematics, https://epubs.siam.org/doi/abs/10.1137/1.9781611970432 (1976).

4. Castillo-Chavez C. and Song B., Dynamical models of Tuberculosis and their application. Math. Biosci. Eng., 1:361-404 (2004).

5. Strogatz, S. Nonlinear dynamics and chaos: with applications to physics, biology, chemistry, and engineering. Boulder: Westview Press (2001)

6. Anderson, R.M., May, R.M., Infectious Diseases of Humans: Dynamics and Control. Oxford University Press, Oxford (1991).

7. Begon, M., Bennett, M., Bowers, R.G., French, N.P., Hazel, S.M., Turner, J., A clarification of transmission terms in hostmicroparasite models: numbers, densities and areas. Epidemiology and Infection., 129:147-53 (2002).

8. De Jong, M.C.M., Diekmann, O., Heesterbeek, J.A.P., How does transmission of infection depend on population size? In: Epidemic models: their structure and relation to data models. Mollison D, ed. Cambridge: Cambridge University Press.84-94 (1995).

9. Diekmann, O., Heesterbeek, J.A.P., Mathematical epidemiology of infectious diseases. Model building, analysis and interpretation. Wiley, New York (2000).

10. Keeling, M.J., Rohani, P., Modeling Infectious Diseases in Humans and Animals. Princeton University Press, Princeton, New Jersey (2008).

11. McCallum, H., Barlow, N., Hone, J., How should pathogen transmission be modelled?. Trends in Ecology and Evolution 16:295-300 (2001).

12. Mugisha, J.Y.T., Ssebuliba, J., Nakakawa, J., Kikawa, C.R., Ssematimba, A., Mathematical modelling of COVID-19 dynamics in Uganda: Using a locally parameterized system to enhance intervention policies. under going peer review (2020).

13. Ssematimba, A., Mugisha, J.Y.T., Luboobi, L.S., Mathematical models for the dynamics of tuberculosis in density-dependent populations: The case of internally displaced people's camps (IDPCs) in Uganda. Journal of Mathematics and Statistics 1:217-24 (2005).

14. https://www.worldometers.info/coronavirus/, accessed on 10/07/2020

15. Nicola, M., Alsafi, Z., Sohrabi, C., Kerwan, A., AlJabir, A., Iosifidis, C., Agha, M., Agha, R. The socio-economic implications of the coronavirus pandemic (COVID-19): A review. International journal of surgery (London, England), 78, 185-193. https://doi.org/10.1016/j.ijsu.2020.04.018 (2020).

16. Gillespie, D.T., (1977). Exact stochastic simulation of coupled chemical reactions. The journal of physical chemistry $81: 2340-61$.

17. Hu, G., Nigmatulina, K., Eckhoff, P., The scaling of contact rates with population density for the infectious disease models. Mathematical Biosciences 244:125 - 134 (2013).

18. Ferguson, N., Cummings, D., Fraser, C.,Cajka, J., Cooley, P., Burke, D., Strategies for mitigating an influenza pandemic. Nature 442:448-452 (2006).

19. Ministry of Health -Uganda. Covid-19 Information Portal: https://covid19.gou.go.ug accessed on 5 August 2020.2020.

20. Wu J.T,. Leung K., Leung G.M. Nowcasting and forecasting the potential domestic and international spread of the 2019-nCoV outbreak originating in Wuhan, China: a modelling study. The Lancet. 2020;395(10225):689-97. doi: 10.1016/S0140-6736(20)302609.

21. Lin Q., Zhao S., Gao D., Lou Y., Yang S., Musa S.S., et al. A conceptual model for the coronavirus disease 2019 (COVID19) outbreak in Wuhan, China with individual reaction and governmental action. International Journal of Infectious Diseases. 2020;93:211-6. doi: 10.1016/j.ijid.2020.02.058.

22. Biryabarema, E. (15 December 2016). "Hatred spills beyond South Sudan along with refugees". Reuters. https://fr.reuters.com/article/us-southsudan-uganda-refugees-idUSKBN1441QU accesed 10 November 2020.

23. Uganda - Refugee Statistics April 2019 - Bidibidi, https://reliefweb.int/report/uganda/uganda-refugee-statistics-april-2019bidibidi accessed 10 November 2020. 
Figures

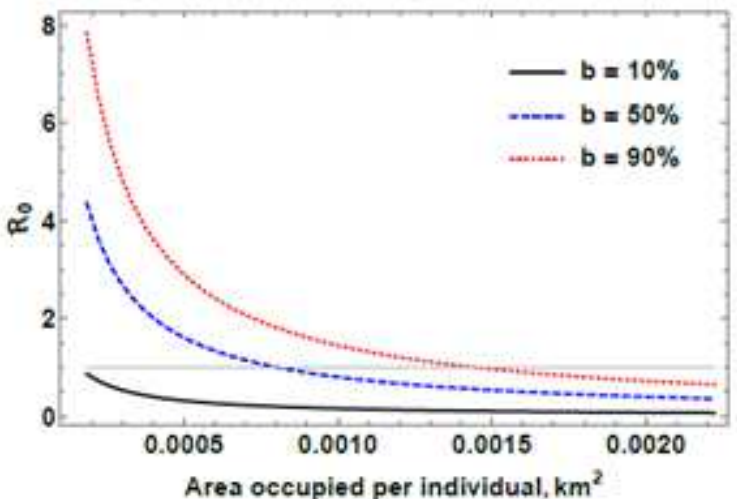

(s)

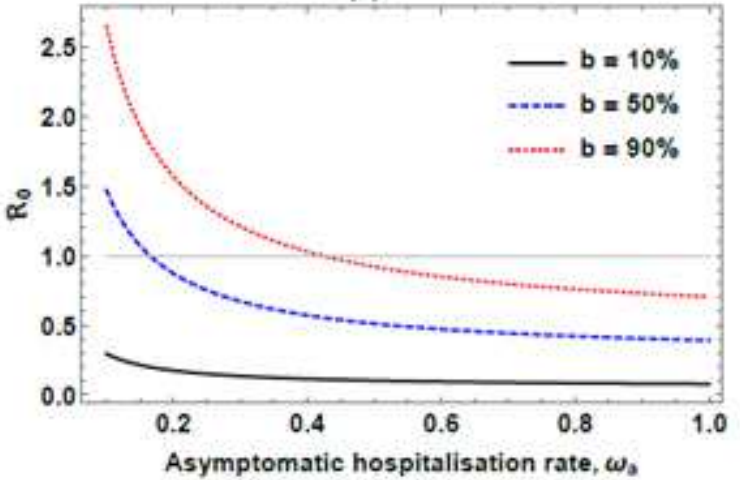

(c)

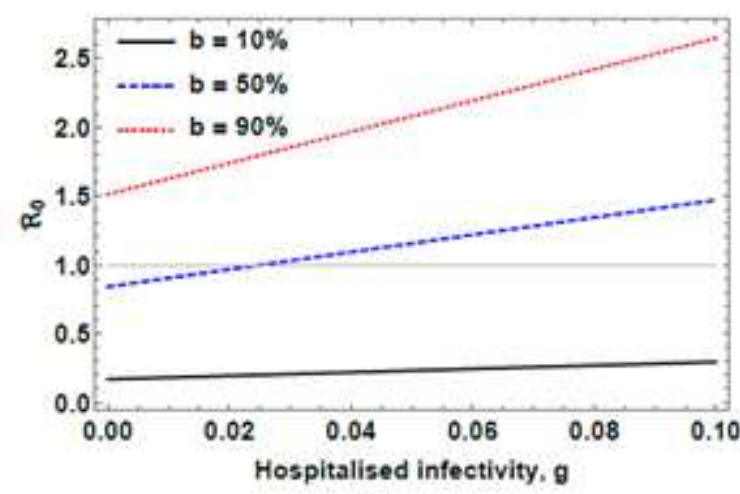

(b)

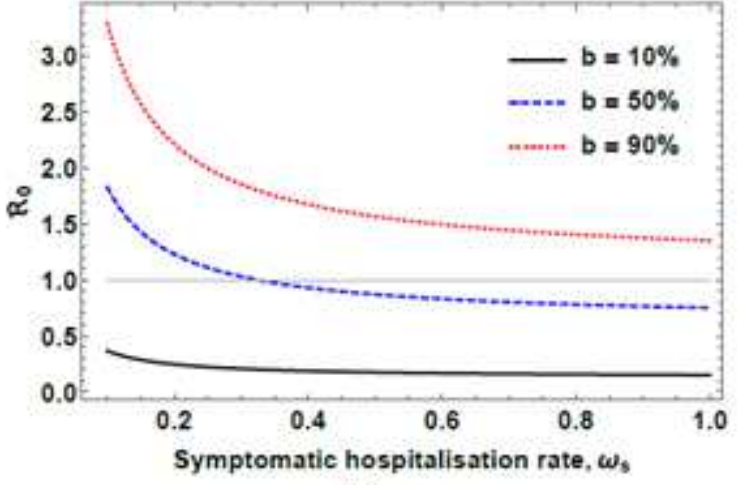

(d)

\section{Figure 1}

Effect of (b) population density, (b) infectivity of hospitalised individuals, (c) hospitalisation rate of asymptomatic infectious individuals and, (d) hospitalisation rate of symptomatic infectious individuals on the basic reproduction number for COVID-19 

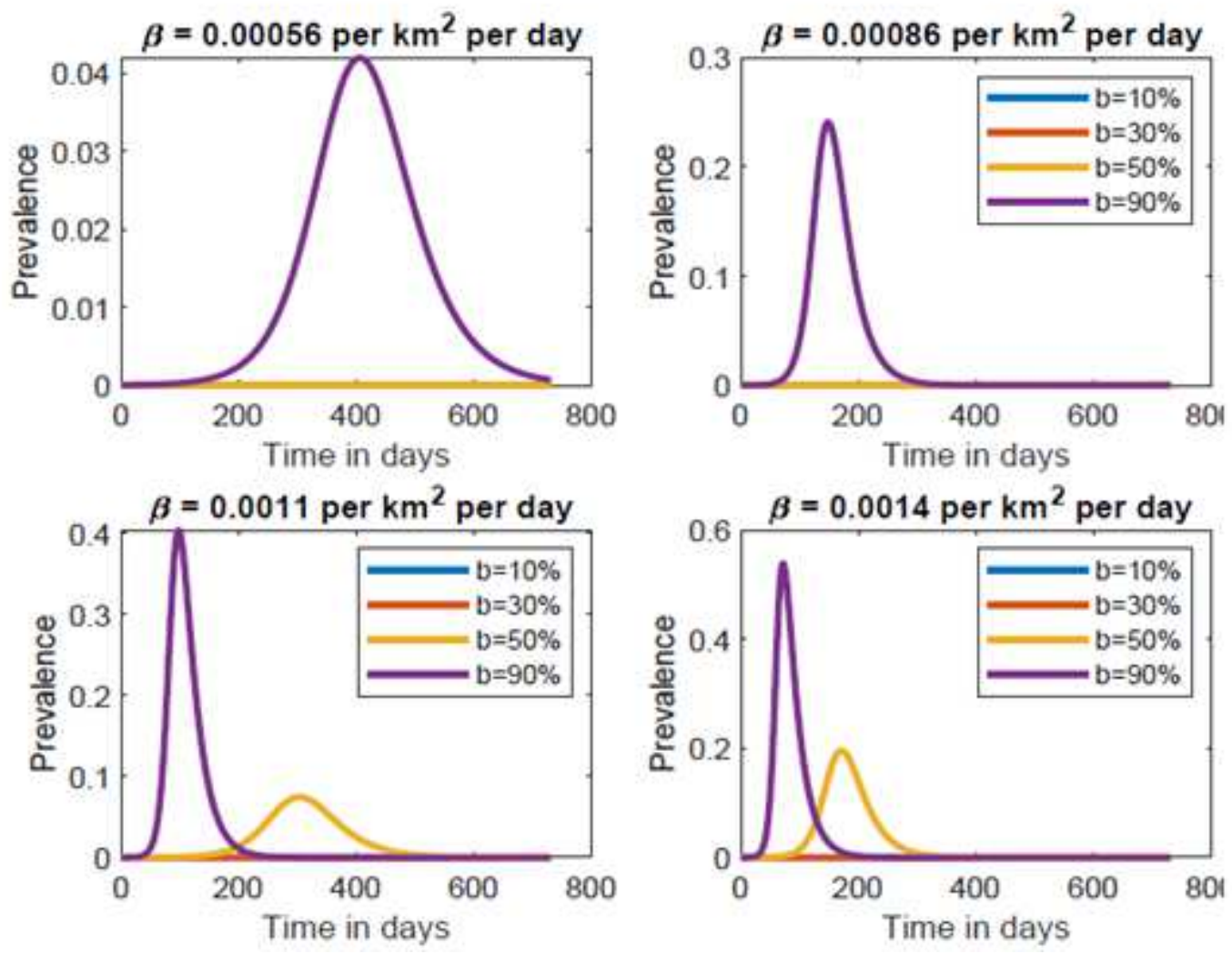

Figure 2

Estimated COVID-19 prevalence for smaller transmission rate per $\mathrm{km} 2$ given different levels of public health interventions 

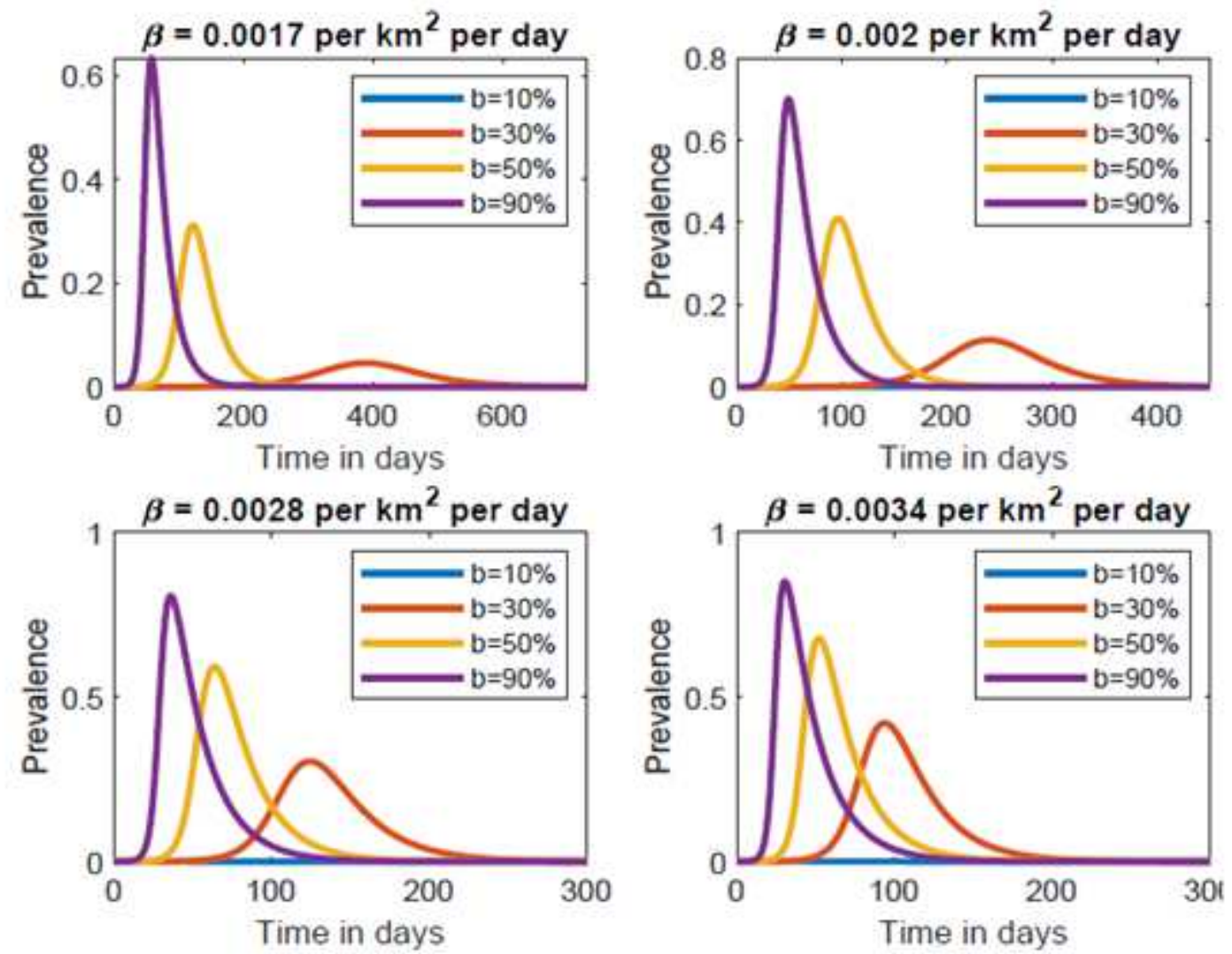

\section{Figure 3}

Estimation of COVID-19 prevalence for slightly higher transmission rates given different levels of adherence to standard operating procedures 